\title{
New classification may assist the development of targeted therapies for treatment-refractory castration-resistant prostate cancer
}

\author{
Ching-Yu Lin ${ }^{1,2}$, Chih-Pin Chuu ${ }^{1,3}$ \\ ${ }^{1}$ Institute of Cellular and System Medicine, National Health Research Institutes, Zhunan, Miaoli; ${ }^{2}$ PhD Program for Cancer Molecular Biology \\ and Drug Discovery, Taipei Medical University, Taipei; ${ }^{3} \mathrm{PhD}$ Program for Aging and Graduate Institute of Basic Medical Science, China Medical \\ University, Taichung \\ Correspondence to: Dr. Chih-Pin Chuu. Room R2-2021, Institute of Cellular and System Medicine, National Health Research Institutes, 35 Keyan \\ Road, Zhunan Town, Miaoli. Email: cpchuu@nhri.org.tw. \\ Provenance and Peer Review: This article is commissioned and reviewed by the Section Editor Dr. Xiao Li (Department of Urology, Jiangsu Cancer \\ Hospital \& Jiangsu Institute of Cancer Research \& Affiliated Cancer Hospital of Nanjing Medical University, Nanjing, China). \\ Comment on: Labrecque MP, Coleman IM, Brown LG, et al. Molecular profiling stratifies diverse phenotypes of treatment-refractory metastatic \\ castration-resistant prostate cancer. J Clin Invest 2019;130:4492-505.
}

Submitted Jan 08, 2020. Accepted for publication Mar 02, 2020.

doi: $10.21037 /$ tau.2020.03.24

View this article at: http://dx.doi.org/10.21037/tau.2020.03.24

For metastatic prostate cancer $(\mathrm{PCa})$, the current standard treatment is androgen deprivation therapy (ADT). However, therapy-resistant tumors, named metastatic castrationresistant prostate cancer (mCRPC), recur in most ADTtreating patients within 3 years and the overall survival time is usually less than 2 years after the relapse $(1,2)$. Docetaxel and two inhibitors targeting androgen receptor (AR) pathway, the abiraterone acetate and enzalutamide, are used for treatment of CRPC. The treatments increased survival as well as reduced pain and serum PSA level (3-6). However, a portion of CRPC patients receiving these drugs develop drug-resistance within a few months $(7,8)$. New therapeutic strategies targeting the treatment-refractory mCRPC are therefore in need. Classification of cancers into subtypes with distinct histological and functional features may assist the development of new targeted therapies.

Labrecque et al. recently used whole-genome sequencing, GSEA (gene set enrichment analysis), and IHC (immunohistochemistry) to analyze 98 tumors obtained at rapid autopsy from $55 \mathrm{PCa}$ patients receiving $\mathrm{ADT}$ in combination with docetaxel, abiraterone or enzalutamide. They provided new classification of mCRPC tumors (9). They demonstrated five different phenotypes based on the expression of AR or NE (neuroendocrine) genes: ARPC (AR-high PCa), ARLPC (AR-low PCa), AMPC (amphicrine tumors composed of cells expressing both $\mathrm{AR}$ and $\mathrm{NE}$ ), DNPC (double-negative $\mathrm{PCa} \mathrm{AR}^{-} / \mathrm{NE}^{-}$), and SCNPC (PCa with small cell or NE expression but without AR activity) (9). They performed RNA-Seq and IHC staining on 18 CRPC LuCaP PDX (patient-derived xenograft) lines to validate the results of the patient specimen analysis and found that the five distinct phenotypes of $\mathrm{mCRPC}$ in PDX lines were accurately segregated based on the AR, NEURO I (SYP, CHGA, SNAP25, SRRM4), and NEURO II (SOX2, POU3F2/BRN2, NKX2-1, and LMO3) gene expression profiles (9). The authors investigated the relationships between these five mCRPC subtypes by examining the progression phenotype of individual mCRPC patient who received a complicated history of treatment. They discovered that mCRPC is a disease continuum, and some subtypes can convert to other subtypes (Figure 1). For example, a small group of DNPC tumor cells have the potential to convert to SCNPC (9). They discovered that the relationship between AR and REST (RE1 silencing transcription factor) were associated with these mCRPC phenotypes. Labrecque et al. analyzed the data from the metastatic tumors in patient as well as the PDX models of $\mathrm{LuCaP}$ cell, they established a transcriptomic signature composed of 26 genes to define the treatment-resistant mCRPC phenotypes (9). 


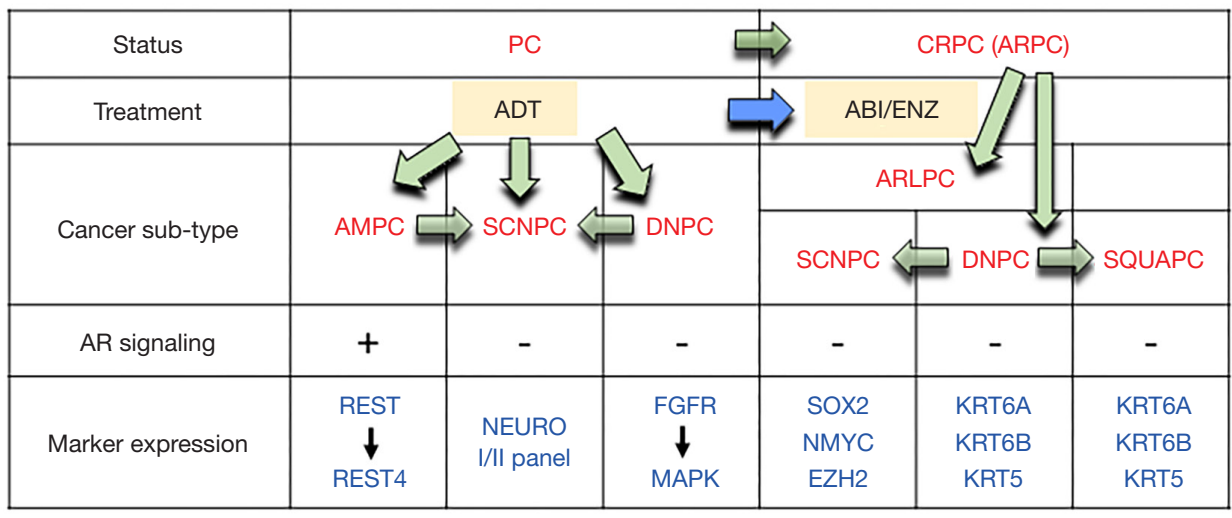

Treatment progress

Transdifferentiation

Figure 1 Progression of treatment-refractory castration-resistant $\mathrm{PCa}$. The proposed mechanism and progression sequence suggested by Labrecque et al. in (9). The PCa cells after receiving ADT can progress to either ARPC, AMPC, SCNPC, or DNPC. ARPC can progress to ARLPC, DNPC, or QUAPC. ADT, androgen deprivation therapy; ABI, abiraterone; ENZ, enzalutamide; PC, hormone-sensitive prostate cancer; ARPC, AR-high prostate cancer; AMPC, amphicrine prostate cancer; SCNPC, small cell or NE prostate cancer; DNPC, doublenegative prostate cancer; ARLPC, AR-low prostate cancer; SQUAPC, squamous prostate cancer.

These findings were really interesting and important. It was very clever to investigate the progression sequence of mCRPC by analyzing the different tumors collected at different time points in mCRPC patients with complicated treatment history. Labrecque et al. found that mCRPC tumors in patients are often heterogenous and are a mixture of different subtypes of mCRPC. Therefore, they can determine which drug or which gene alteration promotes the development of certain subtype of mCRPC tumors. As a result, they confirmed the presence of AMPC cells in patient specimens and in CRPC cell lines (9). They also observed that loss of REST induced the expression of neuroendocrine (NE)-associated genes and drives the conversion of PCa cells to the AMPC phenotype with active AR (9). Additionally, they unearthed that a subtype of mCRPC exhibited features of squamous cell carcinoma and hormone therapy stimulated the transition from ARPC to squamous DNPC at metastatic tumors in PCa patient (9). Currently, there is no ordinary treatment for SCNPC, DNPC, and ARLPC PCa. However, since the authors established a few PDX line from these mCRPC subtypes, a screening of effective compounds and drugs which are capable to suppress the cell proliferation and survival of these drug-resistant mCRPC can be conducted. The transcript panels for tumor classification can be diagnostic or prognostic biomarkers for treatment decision-making. It will also be interesting to use the PDX models to examine if natural compounds which were known to suppress $\mathrm{AR}$ signaling or AR stability, such as EGCG (10) or caffeic acid phenethyl ester (11), may repress the growth of these drugresistant mCRPC xenografts.

The classification of the five subtypes of mCRPC tumors was based on whole-genome sequencing, RNA-Seq and IHC staining analyses. It will be interesting to examine the profile of proteins and metabolites with protein array and LC MS/MS as well as to investigate the profile of tumor associated macrophages (TAMs). It is possible that profiling of proteins and metabolites will further divide the five subtypes of mCRPC into more different subtypes. The difference in profile of protein, metabolite, and TAM among these mCRPC subtypes may allow us to develop the suitable targeted therapy for each patient. However, if patient acquire the heterogenous mCRPC tumor, treatment can be a challenge due to the mixture of different subtypes of mCRPC tumors and the potential existence of cancer stem cell. Biopsy or blood-based biomarkers detection for classification the subtype of mCRPC will therefore be critical to design the personalized medicine for patients with advanced PCa. In conclusion, we believe that the new classification proposed by Labrecque et al. may assist the development of targeted therapies for treatment-refractory mCRPC. 


\section{Acknowledgments}

Funding: This study was supported by intramural grant from National Health Research Institutes and the grant MOST 108-2314-B-400-021 from Ministry of Science and Technology for CPC.

\section{Footnote}

Conflicts of Interest: All authors have completed the ICMJE uniform disclosure form (available at http://dx.doi. org/10.21037/tau.2020.03.24). The authors have no conflicts of interest to declare.

Ethical Statement: The authors are accountable for all aspects of the work in ensuring that questions related to the accuracy or integrity of any part of the work are appropriately investigated and resolved.

Open Access Statement: This is an Open Access article distributed in accordance with the Creative Commons Attribution-NonCommercial-NoDerivs 4.0 International License (CC BY-NC-ND 4.0), which permits the noncommercial replication and distribution of the article with the strict proviso that no changes or edits are made and the original work is properly cited (including links to both the formal publication through the relevant DOI and the license). See: https://creativecommons.org/licenses/by-ncnd/4.0/.

\section{References}

1. Hellerstedt BA, Pienta KJ. The current state of hormonal therapy for prostate cancer. CA Cancer J Clin 2002;52:154-79.

2. Chuu CP, Kokontis JM, Hiipakka RA, et al. Androgens as

Cite this article as: Lin CY, Chuu CP. New classification may assist the development of targeted therapies for treatmentrefractory castration-resistant prostate cancer. Transl Androl Urol 2020;9(2):837-839. doi: 10.21037/tau.2020.03.24 therapy for androgen receptor-positive castration-resistant prostate cancer. J Biomed Sci 2011;18:63.

3. Tannock IF, de Wit R, Berry WR, et al. Docetaxel plus prednisone or mitoxantrone plus prednisone for advanced prostate cancer. N Engl J Med 2004;351:1502-12.

4. Petrylak DP, Tangen CM, Hussain MH, et al. Docetaxel and estramustine compared with mitoxantrone and prednisone for advanced refractory prostate cancer. $\mathrm{N}$ Engl J Med 2004;351:1513-20.

5. de Bono JS, Logothetis CJ, Molina A, et al. Abiraterone and increased survival in metastatic prostate cancer. $\mathrm{N}$ Engl J Med 2011;364:1995-2005.

6. Hussain M, Fizazi K, Saad F, et al. Enzalutamide in Men with Nonmetastatic, Castration-Resistant Prostate Cancer. N Engl J Med 2018;378:2465-74.

7. Ploussard G, Terry S, Maille P, et al. Class III beta-tubulin expression predicts prostate tumor aggressiveness and patient response to docetaxel-based chemotherapy. Cancer Res 2010;70:9253-64.

8. Davies AH, Beltran H, Zoubeidi A. Cellular plasticity and the neuroendocrine phenotype in prostate cancer. Nat Rev Urol 2018;15:271-86.

9. Labrecque MP, Coleman IM, Brown LG, et al. Molecular profiling stratifies diverse phenotypes of treatmentrefractory metastatic castration-resistant prostate cancer. J Clin Invest 2019;130:4492-505.

10. Chuu CP, Chen RY, Kokontis JM, et al. Suppression of androgen receptor signaling and prostate specific antigen expression by (-)-epigallocatechin-3-gallate in different progression stages of $\mathrm{LNCaP}$ prostate cancer cells. Cancer Lett 2009;275:86-92.

11. Kuo YY, Huo C, Lin CY, et al. Caffeic acid phenethyl ester suppresses androgen receptor signaling and stability via inhibition of phosphorylation on Ser81 and Ser213. Cell Commun Signal 2019;17:100. 